\title{
Dor torácica no transtorno de pânico: sintoma somático ou manifestação de doença arterial coronariana?
}

\section{Chest pain and panic disorder: physical symptom or coronary heart disease presentation?}

\author{
Gastão Luiz Fonseca Soares Filho ${ }^{1}$, Alexandre Martins Valença², Antonio Egidio Nardi ${ }^{3}$ \\ 'Psiquiatra, coordenador do Serviço de Interconsulta Psiquiátrica do Hospital Pró-Cardíaco (RJ). Pesquisador do Laboratório de Pânico e Respiração do Instituto de \\ Psiquiatria da Universidade Federal do Rio de Janeiro (UFRJ). \\ ${ }^{2}$ Doutor em Psiquiatria pelo Instituto de Psiquiatria da Universidade Federal do Rio de Janeiro. Professor adjunto de Psiquiatria da Universidade Federal Fluminense (UFF-RJ). \\ Pesquisador do Laboratório de Pânico e Respiração (UFRJ). \\ ${ }^{3}$ Livre-docente e professor adjunto da Faculdade de Medicina do Instituto de Psiquiatria da Universidade Federal do Rio de Janeiro.
}

Recebido: 17/07/2006 - Aceito: 06/09/2006

\begin{abstract}
Resumo
O transtorno do pânico (TP) pertence ao grupo dos transtornos de ansiedade caracterizado por repetidos e inesperados ataques de pânico, nos quais predominam os sintomas somáticos e intensa apreensão relacionada à idéia de perda de controle ou morte iminente. Entre os sintomas somáticos que o paciente pode apresentar, a dor torácica exerce papel preponderante, reforçando a idéia de que ele esteja desenvolvendo problema cardiovascular grave, ameaçador à vida, levando à repetida busca por atendimento em unidades cardiológicas ou outros serviços de emergência. A isquemia miocárdica desenvolve-se quando o fluxo de sangue coronariano se torna inadequado para alcançar as exigências metabólicas miocárdicas e manter a função cardíaca adequada. Sua principal causa é a doença arterial coronariana $(\mathrm{DAC})$ e a mais comum manifestação clínica da isquemia miocárdica é a dor torácica. Este relato de caso ilustra a comorbidade do TP com a DAC, discutindo como lidar com essa complexa situação clínica. O diagnóstico de transtorno de pânico raramente é feito e graves conseqüências podem decorrer disso, inclusive na evolução do transtorno psiquiátrico.
\end{abstract}

Soares Filho, G.L.F et al. / Rev. Psiq. Clin. 34 (2); 97-101, 2007

Palavras-chave: Ansiedade, isquemia miocárdica, diagnóstico, doença arterial coronariana.

\begin{abstract}
Panic disorder is a mental disorder that belongs to the group of the anxiety disorders, characterized by repeated and unexpected panic attacks, in which the somatic symptoms are associated to intense apprehension related to the idea of "loosing control" or an imminent death sensation. Amongst somatic symptoms that patients can present, chest pain plays an important role, reinforcing the idea that the patient is threatened by a serious cardiovascular problem, leading to repeated search for attendance in cardiologic or other emergency rooms. Myocardial ischemia develops when coronary blood flow becomes inadequate to meet the requirements of the myocardium for oxygen and metabolic substrates to maintain adequate cardiac function. Coronary stenosis is considered the main cause of myocardial ischemia and its most common clinical manifestation is chest pain. This case report illustrates panic disorder co-occurring with coronary heart disease, discussing how to deal with this complex clinical situation. The diagnosis of panic disorder seldom is made and serious consequences can elapse, including the course of the psychiatric disorder.
\end{abstract}

Soares Filho, G.L.F et al. / Rev. Psiq. Clín. 34 (2); 97-101, 2007

Key-words: anxiety, myocardial ischemia, diagnoses, coronary heart disease.

Endereço para correspondência: Rua Raul Pompéia, 141, ap. 204 - 22080-000 - Rio de Janeiro, RJ. Fones: (21) 2227-5296/9695-9008. E-mail: galufo@gmail.com 


\section{Introdução}

O transtorno do pânico (TP) é caracterizado pela ocorrência espontânea e inexplicável de ataques de pânico, que são períodos de intenso medo, podendo variar desde diversos ataques ao dia até poucos no curso de um ano. A expressão desse medo é dada por sintomas somáticos e cognitivos como taquicardia, sudorese, falta de ar, desrealização, despersonalização, medo de enlouquecer ou morrer (APA, 2002). É freqüentemente acompanhado por agorafobia, que é o temor de se encontrar sozinho em lugares públicos, os quais tenham como característica a dificuldade de uma saída rápida, caso surja novo ataque de pânico.

Como chama a atenção Fleet et al. (2000), 6 dos 13 sintomas dos critérios diagnósticos do transtorno de pânico são também achados freqüentes em doenças do coração, como dor torácica, palpitações, sudorese, sensação de asfixia, sufocação e ondas de calor. O indivíduo acometido por ataque de pânico freqüentemente acredita estar apresentando um quadro orgânico grave, como infarto agudo do miocárdio, e o primeiro atendimento é comumente realizado em unidades de emergências cardiológicas. É compreensível que indivíduos com transtorno de pânico utilizem os serviços de emergência com muita frequiência, gerando alto custo de assistência médica.

Ador torácica é um sintoma comum na prática médica, sendo a queixa principal de muitos pacientes em atendimento médico primário. É também um dos principais sintomas da isquemia miocárdica, presente na doença arterial coronariana (DAC), devendo ser ressaltado que a possibilidade de esta estar associada ao TP é um fato. Em um estudo que observou pacientes atendidos em departamentos de emergência com sintoma de dor torácica e avaliados por meio de teste ergométrico ou arteriografia coronariana, uma entrevista psiquiátrica, prévia à cardiológica, revelou dados significativos. Dos 1.364 pacientes com dor torácica, 411 apresentavam TP e destes, 306 não tinham diagnóstico de DAC. Por outro lado, dos 1.364 pacientes, apenas 248 apresentavam DAC sem TP. Algumas conclusões importantes podem ser tiradas desse estudo. Dentre aqueles que chegaram à emergência com dor torácica, $30,1 \%$ apresentavam TP e $22,4 \%$ tinham TP sem DAC. Analisando apenas aqueles com TP, 74,4\% (306/411) não apresentavam DAC. Embora tenha sido encontrada uma grande proporção de pacientes com TP sem DAC, ainda assim é relevante o achado de que aproximadamente $26 \%$ (105/411) dos pacientes com TP eram também portadores de DAC (Lynch e Galbraith, 2003).

A dor torácica deve ser sempre investigada com atenção. O foco principal de um atendimento sistematizado é a identificação precoce do risco de eventos ameaçadores à vida. Isto inclui a rápida triagem de pacientes com alta probabilidade de infarto, a estratificação quanto à presença de isquemia miocárdica e o diagnóstico de doenças cardiovasculares não coronarianas, doenças pulmonares e do trato digestivo de alto risco.

\section{Relato de caso}

S., sexo feminino, 55 anos, casada, ensino médio completo, do lar. Apresentou em dezembro de 2001 quadro abrupto de dor torácica opressiva, retroesternal, acompanhada de falta de ar, dormência nas extremidades dos membros inferiores e superiores, além de angústia intensa e sensação de morte iminente. Procurou emergência cardiológica onde foi submetida a exame clínico e eletrocardiograma de repouso, recebendo alta após ser informada de que "não estava infartando". Cinco dias após, a paciente apresentou nova crise de ansiedade, com características semelhantes à primeira. Foi a outro serviço de emergência, ainda acreditando estar apresentando um ataque cardíaco. Após nova avaliação clínica, foi descartada a existência de evento coronariano agudo, levantando a hipótese de ataque de pânico. Foi, então, recomendado à paciente que procurasse atendimento em serviço de psiquiatria, mas não houve prescrição de qualquer medicação.

No início de 2002, a paciente se consultou com médico clínico, que realizou exames de sangue, ecocardiograma e novo eletrocardiograma de repouso. Após constatar que os resultados eram normais, prescreveu paroxetina $10 \mathrm{mg} /$ dia. No dia seguinte à primeira tomada do medicamento, a paciente informou que experimentou quadro de "angústia descomunal", associada a dor torácica, falta de ar e dormência de extremidades, interrompendo o uso da medicação, que considerava ter sido a causa de sua crise de ansiedade. Relata que 0 ataque que se seguiu à ingesta de uma dose de $10 \mathrm{mg}$ de paroxetina foi o pior de todos. "Não gosto nem de lembrar", disse. "A dormência, inicialmente nos pés e nas mãos, começou a subir pelas pernas e pelos braços e eu achava que se não parasse de subir, eu ia morrer. Eu tentava respirar e 0 ar não entrava. Parecia que havia uma pilha de tijolos no meu peito". Após esse dia, diz que passou a sentir "muito medo, de tudo, principalmente de tomar remédios”. Não saía de casa para nada, e, para usar o banheiro, tinha de deixar a porta aberta.

Posteriormente, S. passou a experimentar episódios de dor no peito e falta de ar quase diários. Conta que enquanto não apresentava as "crises", experimentava forte ansiedade com a possibilidade de que nova "crise" viesse a acontecer, ansiedade esta que durava a maior parte do dia. Passou a evitar lugares onde fosse difícil obter ajuda. Embora já houvesse tido informações especializadas de que não era portadora de doença do coração, ainda temia os episódios de dor no peito e falta de ar, sempre acompanhados de um medo de morte intenso. Seguiu vários tratamentos à base de antidepressivos e ansiolíticos, sem regularidade em relação ao uso da medicação e às visitas médicas.

Quando procurou o Laboratório de Pânico e Respiração do Instituto de Psiquiatria (UFRJ), em março de 2005, a paciente se queixava de ainda apresentar episódios de dor torácica e falta de ar que geralmente eram 
desencadeados por situações de estresse. Seus deslocamentos pela cidade se restringiam às ruas e avenidas sem túneis e sem favelas próximas. Saía de casa apenas para ir ao médico e ao fisioterapeuta. Embora gostasse de cinema e teatro, estava evitando esses locais, com medo de "ter uma crise e passar um vexame". Na maior parte do seu dia, convivia com sensação de insegurança e ansiedade. Os sintomas somáticos marcantes, como falta de ar e dor torácica, surgiam quando se via diante de tarefas as quais não se considerava em condições de enfrentar, como ir a bancos e administrar o dinheiro da família.

Tabagista de duas carteiras de cigarro/dia, portadora de dislipidemia, S. perdera o irmão quando este tinha 50 anos, vítima de infarto agudo do miocárdio, e seu pai aos 70 anos, de "problemas respiratórios e cardíacos". Sua mãe e seu filho, com 36 anos, estão vivos e saudáveis. Foi realizada avaliação por meio de diagnóstico estruturado - MINI (Mini International Neuropsychiatric Interview, versão 4.4), sendo identificado o diagnóstico de transtorno de pânico atual com agorafobia. A paciente confirmou a presença dos seguintes sintomas, com intensidades variadas: dificuldade para respirar; sensação de sufocamento; dor no peito; vertigens; medo de morrer; dormência nas mãos; e sensação gelada no corpo. Ao exame clínico, mostrava pressão arterial e freqüência cardíaca nos limites da normalidade.

Foi prescrito à paciente o uso de fluoxetina $20 \mathrm{mg} / \mathrm{dia}$ e clonazepam $0,5 \mathrm{mg}$ pela manhã e $1,0 \mathrm{mg}$ à noite, sendo solicitados exames laboratoriais, eletrocardiograma (ECG) de repouso e teste ergométrico.

No retorno, a paciente se dizia ainda preocupada com a possibilidade de novos ataques, saindo de casa sempre acompanhada, mas relatava que já não faltava aos seus compromissos e que o nível de ansiedade havia se reduzido. Não conseguiu tomar a fluoxetina além de $13 \mathrm{mg} / \mathrm{dia}$, pois ainda sentia muito medo. Seus exames laboratoriais foram normais, inclusive as taxas de colesterol, porém o ECG de repouso mostrava uma alteração de repolarização na parede inferior e o teste ergométrico era positivo para doença isquêmica, na mesma parede inferior. Diante do resultado, foi sugerido à paciente que procurasse seu cardiologista, pois poderia ser necessário prosseguir na investigação para elucidação do quadro, o que a deixou ainda mais ansiosa.

Cerca de quinze dias depois, a paciente retornou trazendo o resultado da cineangiocoronariografia solicitada por seu médico, cujo laudo era normal, descartando doença coronariana. Relatava uma progressiva melhora dos sintomas fóbicos, já não evitando tarefa externa alguma dentro dos limites anteriormente descritos, mas ainda necessitando de companhia. Dizia já estar conseguindo tomar $18 \mathrm{mg} /$ dia da fluoxetina. Nesta ocasião, foi encaminhada à psicoterapia do tipo cognitivo-comportamental, sendo mantido o tratamento com fluoxetina 20 $\mathrm{mg}$ por dia de modo regular, além do clonazepam.

\section{Discussão}

O caso clínico anterior é exemplar no que tange à complexidade da apresentação clínica e à abordagem do TP.

O início do problema da paciente se dá a partir de um ataque de pânico não diagnosticado, com intensos sintomas somáticos do espectro cardiovascular. Pacientes com ataques de pânico associados à dor torácica apresentam grande urgência de atendimento, e essa associação não é incomum. Pacientes com TP freqüientemente se apresentam à sala de emergência com queixas de dor torácica e palpitação. Dor torácica em pacientes portadores de TP tem prevalência de 25\% a 57\% (Carter et al., 1994; Fleet et al., 1996). Como procuram emergências clínicas, o sintoma de dor torácica invariavelmente é investigado sob a ótica da doença arterial coronariana (DAC). Já foi demonstrado (Mayou, 1998) que pacientes na meia-idade, com história familiar importante para cardiopatia, tendem a apresentar dor torácica mais frequientemente. Uma avaliação restrita à área cardiocirculatória por parte do médico de emergência pode desencadear todo o processo investigativo diagnóstico ou mesmo o início de tratamento com medicação cardiovascular. Esses pacientes passam a ter a vida limitada, sem alívio dos sintomas e da ansiedade.

No entanto, é a falta do diagnóstico e tratamento corretos do TP que leva à busca repetida de atendimento por médico clínico, quando da ocorrência de novo ataque de pânico (Lynch e Galbraith, 2003), e esse diagnóstico raramente é realizado, quando se trata de paciente chegando à sala de emergência com dor torácica. Em conseqüência dessa "cegueira" diagnóstica, o paciente recebe tratamento inadequado para o $\mathrm{TP}$, ocorrendo conseqüente cronificação dos sintomas, limitação das atividades e redução da qualidade de vida, além de uso excessivo e inadequado dos recursos médicos.

Estudo realizado em departamento de emergência (Fleet et al., 1998), com pacientes apresentando dor torácica, observou que entre os que apresentavam critérios diagnósticos para TP, apenas $2 \%$ tiveram o diagnóstico correto no momento da chegada. Em outro trabalho (Wulsin et al., 1988), também realizado em departamento de emergência, o diagnóstico de transtorno psiquiátrico foi feito em apenas 1 de 30 pacientes com TP, mostrando falta de diagnóstico em $97 \%$ dos atendimentos. Investigadores têm buscado medidas para minimizar a dificuldade diagnóstica apresentada pelos profissionais que trabalham em serviços de emergência. Num estudo retrospectivo em que foram revistos os trabalhos realizados entre 1970 e 2001 (Huffman e Pollack, 2003), investigando a prevalência de TP em pacientes com dor torácica, cinco variáveis mostraram alta taxa de correlação estatística: ausência de doença coronariana; dor torácica atípica; sexo feminino; faixa etária mais jovem; e altas taxas de ansiedade auto-relatadas. 
$\mathrm{Na}$ falta de um diagnóstico psiquiátrico adequado, seria de se esperar que a informação dada ao paciente, de que seus sintomas não se devem a um evento cardíaco agudo, fosse suficiente para gerar alívio dos sintomas e interrupção dos ataques de pânico. No entanto, a literatura tem demonstrado que investigações negativas, por meio de avaliação clínica (Mayou et al., 1994) ou teste ergométrico (Channer et al., 1987), foram insuficientes para a supressão dos sintomas de pânico, das altas taxas de re-utilização dos recursos médicos e do alto grau de dano no status funcional. Nem mesmo quando os pacientes são submetidos à investigação invasiva, por meio de coronariografia, os resultados normais são suficientes para gerar tranquiilidade e ausência de sintomas. Estudos de seguimento mostram que, independentemente do tempo de doença, os sintomas persistem enquanto o diagnóstico psiquiátrico não é realizado. Bass et al. (1983) acompanharam por um ano pacientes com dor torácica e coronariografia normal. Destes, $41 \%$ seguiam com queixas de dor torácica, $63 \%$ ainda se consultavam com médicos não psiquiatras e $24 \%$ eram incapazes de trabalhar, em função da dor torácica. Um segundo estudo de seguimento (Potts e Bass, 1995) acompanhou pacientes em condições semelhantes por 11 anos e constatou $74 \%$ desses pacientes ainda se queixando de dor torácica.

A paciente do caso relatado, após "peregrinar" por emergências, foi atendida por um psiquiatra que prescreveu um inibidor seletivo de recaptação de serotonina (ISRS). Talvez pela piora inicial, possível ao se iniciar um antidepressivo ISRS em pacientes com TP (Starcevic, 2005), na manhã seguinte ao uso do antidepressivo, a paciente apresentou um novo episódio de ataque de pânico, intenso e com rica manifestação ansiosa. Durante seus ataques de pânico, os sintomas somáticos, como dor torácica, parestesias, taquicardia, eram acompanhados por sentimentos aterrorizantes que a levavam à convicção de morte iminente. Esses sentimentos se devem a uma característica dos pacientes com TP de terem um modo particular e catastrófico de interpretar os sintomas corporais, o que pode estar relacionado ao desencadeamento ou à manutenção dos ataques de pânico. Talvez isso explique aquele que se seguiu à ingestão da primeira dose do ISRS. É possível que o medicamento tenha produzido algum efeito adverso que, mesmo leve, tenha sido interpretado de maneira catastrófica pela paciente, gerando um novo ataque. Como o retardo no início dos efeitos terapêuticos dos antidepressivos deixa o paciente desprotegido por semanas, pode eventualmente ser necessária a associação de um benzodiazepínico, principalmente quando intensa ansiedade está presente.

Em relação à abordagem diagnóstica da dor torácica, alguns comentários se fazem pertinentes. A paciente apresentava dor torácica que pode ser classificada como típica para DAC: opressiva, como um peso sobre o peito, mal localizada, difusa em toda a região retroesternal.
Também relatava fatores de risco importantes para doença coronariana, como história familiar, tabagismo e dislipidemia. Se, por um lado, a dor torácica é um sintoma freqüente nos pacientes portadores de TP sem DAC, nada impede que pacientes portadores de TP também apresentem DAC, contexto no qual a avaliação da dor torácica se torna bastante complexa. Essa associação foi mostrada pela primeira vez por Beitman et al. (1987), que verificaram prevalência de $57 \%$ de TP em pacientes cardiopatas. Posteriormente, Basha et al. (1989) também examinaram a prevalência de transtorno de pânico em pacientes com DAC, que apresentavam dores torácicas típica e atípica. Entre eles, 27\% preenchiam critérios para TP pelo DSM-III, todos com dor torácica típica. Em contraste, nenhum daqueles que apresentavam dor típica teve diagnóstico confirmado de TP. Os achados sugerem que o TP é prevalente entre os pacientes com DAC, embora possa ser mais comum naqueles que apresentam dor atípica.

A paciente foi submetida à investigação diagnóstica com exames laboratoriais e ECG de repouso. Sabe-se, no entanto, que o ECG de repouso tem sensibilidade ao redor de $40 \%$ para o diagnóstico da DAC na sua fase aguda, isto é, em presença de isquemia miocárdica. Fora dos períodos críticos, essa sensibilidade é ainda mais baixa, sendo necessária a realização de teste provocativo, e o ECG de esforço é o método de eleição, só perdendo em sensibilidade para a cintilografia miocárdica de esforço (O’Rouke et al., 2004). O teste ergométrico solicitado posteriormente sugeria a presença de isquemia de esforço induzida na parede inferior, o que motivou a indicação de que a paciente procurasse um cardiologista. Ao retornar, a paciente trazia o laudo de estudo angiográfico coronariano, cujo resultado mostrava artérias livres de obstruções, descartando DAC. Tal achado não é incomum, sendo de $23 \%$ a taxa de ECG de esforço com resultados falso-positivos (O'Rouke et al., 2004). Como mostra a literatura mencionada anteriormente, as informações dadas à paciente de que não era portadora de DAC não foram suficientes para eliminar o medo de novos ataques de pânico. Ela seguiu em tratamento com ISRS e BZD, e, embora com menos ansiedade, persistiam os sintomas evitativos agorafóbicos, sendo encaminhada à terapia cognitivo-comportamental (TCC).

\section{Conclusões}

A dor torácica é uma queixa comum entre os pacientes portadores de transtorno de pânico (TP), assim como entre aqueles que procuram atendimento em serviços de emergência.

Pacientes com queixa de dor torácica que procuram atendimento de emergência podem apresentar graves quadros orgânicos das esferas cardiovascular, pulmonar ou gastrintestinal, mas também podem ser portadores de TP, comórbido ou não aos transtornos orgânicos. 
Este caso ilustra bem como os sintomas do transtorno de pânico se confundem com aqueles apresentados em sérias condições médicas, como a doença arterial coronariana (DAC). É de extrema importância uma boa avaliação clínica e psiquiátrica em pacientes com dor torácica que se apresentam em serviços de emergência clínica e cardiológica. O diagnóstico e o tratamento precoces, tanto da DAC quanto do TP, podem diminuir as complicações relacionadas a essas condições, bem como melhorar a qualidade de vida desses pacientes.

É importante que sejam criados critérios e métodos diagnósticos específicos para o TP associado à dor torácica. Pacientes com dor torácica que procuram serviços de emergência devem ser sistematicamente investigados em relação à possibilidade de TP.

É fundamental que tanto psiquiatras quanto cardiologistas, especialmente aqueles que trabalham em serviços de emergência, estejam atentos à complexa inter-relação que envolve o transtorno de pânico e o sintoma de dor torácica.

\section{Referências}

Associação Psiquiátrica Americana. Manual diagnóstico e estatístico de transtornos mentais - DSM-IV-TR. Trad. Claudia Dornelles. $4^{\text {a }}$ ed. rev. Artmed, Porto Alegre, pp. 419-431, 2002.

Basha, I. et al. Atypical angina in patients with coronary artery disease suggests panic disorder. Int J Psychiatry Med 19: 341-346, 1989.

Bass, C.; Wade, C.; Hand, D.; Jackson, G. Patients with angina with normal and near normal coronary arteries: clinical and psychosocial state 12 months after angiography. BMJ (Clinical Research Edition) 287:1505-8, 1983.
Beitman, B.D.; Basha, I.; Flaker, G. Atypical or nonanginal chest pain. Panic disorder or coronary artery disease? Arch Intern Med 147:1548-1552, 1987.

Carter, C.; Maddock, R.; Zoglio, M.; Lutrin, C.; Jella, S.; Amsterdam, E. Panic disorder and chest pain: a study of cardiac stress scintigraphy patients. Am J Cardiol 74: 296-298, 1994.

Channer, K.S. et al. Failure of a negative exercise test to reassure patients with chest pain. Q J Med 240:315-322, 1987.

Fleet, R.C.; Dupuis, G.; Marchand, A.; Burelle, D.; Arsenault, A.; Beitman, B.D. Panic disorder in emergency department chest pain patients: prevalence, comorbidity, suicidal ideation and physical recognition. Am J Med 101: 371-380, 1996.

Fleet, R.P.; Dupuis, G.; Marchand, A.; Beitman, B.D. Panic disorder in coronary artery disease patients with noncardiac chest pain. Journal of Psychosomatic Research 44 (1): 81-90, 1998.

Fleet, R.P.; Lavoiea, K.; Beitmane, B.D. Is panic disorder associated with coronary artery disease? A critical review of the literature. Journal of Psychosomatic Research 48, 347-356, 2000.

Huffman, J.C.; Pollack, M.H. Predicting panic disorder among patients with chest pain: an analysis of the literature. Psychosomatics 44(3): 222-36, 2003.

Lynch, P.; Galbraith, K.M. Panic in the emergency room. Can J Psychiatry 48:361-366, 2003.

Mayou, R.A. et al. Non-cardiac chest pain and benign palpitations in the cardiac clinic. Br Heart J 72:548-553, 1994.

Mayou, R.A. Chest pain, palpitations and panic. Journal of Psychosomatic Research 44(1): 53-70, 1998.

O'Rourke, L.A.; O'Gara, P.; Douglas, J.S. Coronary blood flow and myocardial ischemia. In: Fuster, V.; Wayne, A. R.; O'Rourke, L.A., Robert, A. Hurst's the heart. 11th ed. McGraw-Hill, New York, pp. 1466-1493, 2004.

Potts, S.G.; Bass, C.M. Psychological morbidity in patients with chest pain and normal or near-normal coronary arteries: a long-term follow-up study. Psychol Med 25:339-47, 1995.

Starcevic, V. Panic disorder with and without agoraphobia. In: Anxiety disorders in adults: a clinical guide. 1st ed. Oxford University Press, New York, pp. 14-101, 2005

Wulsin, L.R.; Hillard, J.R.; Geier, P.; Hissa, D.; Rouan, G.W. Screening emergency room patients with atypical chest pain for depression and panic disorder. Int J Psychiatry Med 18:315-23, 1988. 